H I G H L I G H TS

YEAST GENETICS

\section{What makes daughters different}

In yeast, mothers and daughters are genetically identical — they refer to the products of mitotic cell division. But there are also some important differences, which makes yeast a great system for understanding how cell division can give rise to different cell fates - an important general phenomenon. By studying this process in Saccharomyces cerevisiae, ColmanLerner et al. have found eight genes that are expressed only in daughter cells, and they speculate that the regulation of these genes might constitute a new checkpoint, at the very end of the cell cycle.

Cell division in S. cerevisiae begins when a cell produces a small bud, which grows until the cell undergoes mitosis, and ends when the newly formed daughter cell separates from the mother cell. A well-known difference between mother and daughter cells is that mothers retain a 'bud scar', made of residual cell-wall material, whereas daughters have a less obvious 'birth scar'. This is thought to reflect the way the cell wall separates in the final stages of cell division. One of the genes known to be involved in this process is CTS1, which encodes a cellwall-degrading enzyme.

Colman-Lerner and colleagues began their study by looking at CTS1 transcription and found that it is expressed only in daughter cells. To see if there might be other genes that are transcribed only in daughters, they analysed published microarray data and found 26 genes that are coexpressed with CTS1 under a variety of conditions. They found that seven of these genes are indeed daughter specific - a neat demonstration of how microarray data can be applied to problems for which the microarray experiment was not originally designed.

Of the eight daughter-specific genes (including CTS1), four are implicated in cell-wall degradation, which suggests that the cell wall is degraded mainly from the daughter's side and might explain why a prominent bud scar forms only on the mother cell. Regulating cell-wall degradation in this way might help to ensure that the cell wall that connects the mother and bud does not break down until the underlying cell membrane has fully separated - a cytokinesis checkpoint. Most significantly, the authors build up a model for the regulation of the daughter-specific genes in which all eight genes are regulated by a trio of proteins - the transcriptional regulator Ace2, the protein kinase Cbk1 and its binding partner Mob2. What's more, all three genes are conserved in other organisms, so Colman-Lerner et al. might well be on the trail of a widespread mechanism for asymmetric cell division.

Mark Patterson

(2) References and links ORIGINAL RESEARCH PAPER Colman-Lerner, A. et al. Yeast Cbk1 and Mob2 activate daughterspecific genetic programs to induce asymmetric cell fates. Cell 107, 739-750 (2001) WEB SITES

WEB SITES

http://www.els.net

Budding

The Molecular Sciences Institute:

http://www.molsci.org

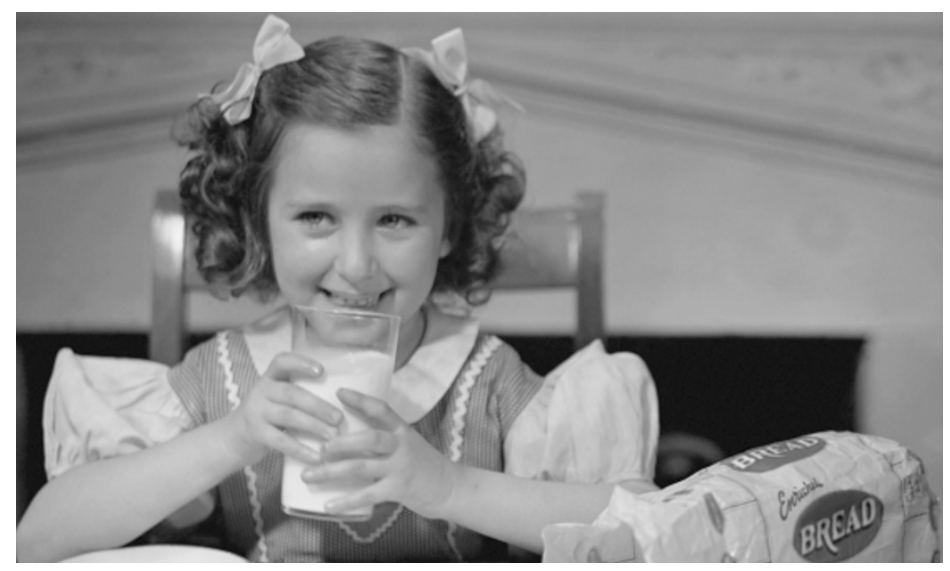

\section{IN BRIEF}

\section{GENOMICS}

The DNA sequence and comparative analysis of human chromosome 20.

Deloukas, P. et al. Nature 414, 865-871 (2001)

This paper reports the completion of the sequence of the euchromatic portion of human chromosome 20 , and the analysis and annotation of the sequence. The value of having finished sequence available is highlighted by the finding of several discrepancies between these results and those reported last year in the draft human sequence analysis. The authors boosted their gene-prediction and gene-annotation analyses by using whole-genome shotgun data from two other vertebrates, the mouse and the pufferfish.

\section{EPIGENETICS}

Differentially methylated forms of histone $\mathrm{H} 3$ show unique association patterns with inactive X chromosomes.

Boggs, B. A. et al. Nature Genet. 30, 73-76 (2002)

Histone $\mathrm{H} 3$ lysine 9 methylation is an epigenetic imprint of facultative heterochromatin.

Peters, A. H. F. M. et al. Nature Genet. 30, 77-80 (2002)

Mammalian X-chromosome inactivation is an ideal model for studying the link between histone methylation and epigenetic gene regulation, as investigated in these studies. Both teams used antibodies against the methylated form of Lys 9 on histone H3 (H3-Lys9) to show that this methylated residue is enriched in heterochromatin on inactive human $\mathrm{X}$ chromosomes. As it is retained during mitosis and chromosome condensation, this methylated residue might be a stably propagated epigenetic mark for the inactive $\mathrm{X}$. As Peters et al. found, it is also retained in the absence of the histone methyltransferase, Suv39, in female mouse cells, which abolished H3-Lys9 methylation in constitutive but not in facultative heterochromatin, indicating that a Suv39independent pathway regulates $\mathrm{H} 3$-Lys9 methylation in facultative heterochromatin.

\section{COMPLEX DISEASE}

Independent genome-wide scans identify a chromosome 18 quantitative-trait locus influencing dyslexia.

Fisher, S. E. et al. Nature Genet. 30, 86-91 (2002)

For conditions such as dyslexia, which have an important genetic component, but are also highly heterogeneous, it is very difficult to identify the key genetic loci. Fisher et al. have tackled this problem with a QTL-based approach in which individuals are assessed using quantitative criteria related to word recognition. In a genome-wide scan, they find evidence to support previous reports of a dyslexia locus on chromosome 6, but also find new QTL, in particular one on chromosome 18. 